\section{Is SAPHO Syndrome Linked to PASH Syndrome and Hidradenitis Suppurativa by Nicastrin Mutation? A Case Report}

\section{To the Editor:}

The SAPHO syndrome is a group of disorders that manifest as synovitis, acne, pustulosis, hyperostosis, and osteitis. Of these, acne damage can present as acne conglobata, acne fulminans, or hidradenitis suppurativa (HS). Studies have shown that some patients with HS or pyoderma gangrenosum, acne, and HS (PASH) syndrome have mutations in gamma-secretase, with alterations in its nicastrin (NCSTN) subunit being especially common ${ }^{1,2}$. Currently, the pathogenic genes of SAPHO syndrome are not yet known. Here, we describe the detection of a mutation in the NCSTN subunit of gamma-secretase in a sporadic patient with SAPHO syndrome presenting with HS.

A 44-year-old Chinese Han male patient with a history of pain in the elbows, knees, sternoclavicular joint, and sacroiliac joint for $>10$ years presented with acne and HS in the face and trunk within the last 4 years. Oral isotretinoin treatment was ineffective. The physical examination revealed inflammatory papules and depressed scars on the face. Inflammatory papules, nodules, and cysts accompanied by scarring were found on the back of the neck, back, axilla, and buttocks (Figure 1). Results of the tenderness tests of both sternoclavicular joints, left elbow, and left knee were positive. A compression test of the sacroiliac joint was positive. The computed tomography scan showed erosion-like changes in the sacroiliac joint (Figure 2). Histological examination of the skin biopsy showed dense neutrophilic inflammation. His parents and 1 younger sister had no similar lesions or symptoms. The patient was diagnosed as having SAPHO syndrome.

Our study was conducted in accordance with the principles of the Declaration of Helsinki. After approval was obtained from the Ethics Committee of the Institute of Dermatology of the Chinese Academy of Medical Sciences (no. 2014-KY-017) and informed consent was signed by the participants, peripheral blood samples were collected. Genomic DNA was extracted; the exomes of this patient were subjected to exome sequencing and then to Sanger sequencing. Genetic examination revealed that he had a single-nucleotide deletion mutation of a cytosine at position 278 (c.278delC) of the nicastrin gene (GenBank NM_015331.2), leading to a heterozygous frameshift mutation (p.P93LfsX15; Figure 3). Unaffected members of this pedigree and 100 controls showed no pathogenic variant.

The SAPHO syndrome was first proposed by Chamot, et al in 1987 to describe a group of chronic aseptic inflammatory disorders involving the skin and joints ${ }^{3}$. Currently, this syndrome has many diagnostic criteria, but the criteria proposed by Kahn and Khan in 1994 are most often mentioned, as confirmed by Marzano, et $a l^{4}$. The patient in our current case study had acne and HS accompanied by systemic polyarthritis and changes in the sacroiliac joint, and thus was diagnosed as having SAPHO syndrome.

Pathogenic genes of the SAPHO syndrome are not yet known, although early studies reported several susceptibility genes such as proline-serine-threonine phosphatase-interacting protein 2 and murine double minute- 2 might be involved ${ }^{5,6}$. More than 25 papers in the literature have reported the relationship between HS and NCSTN mutations. Additionally, several gene
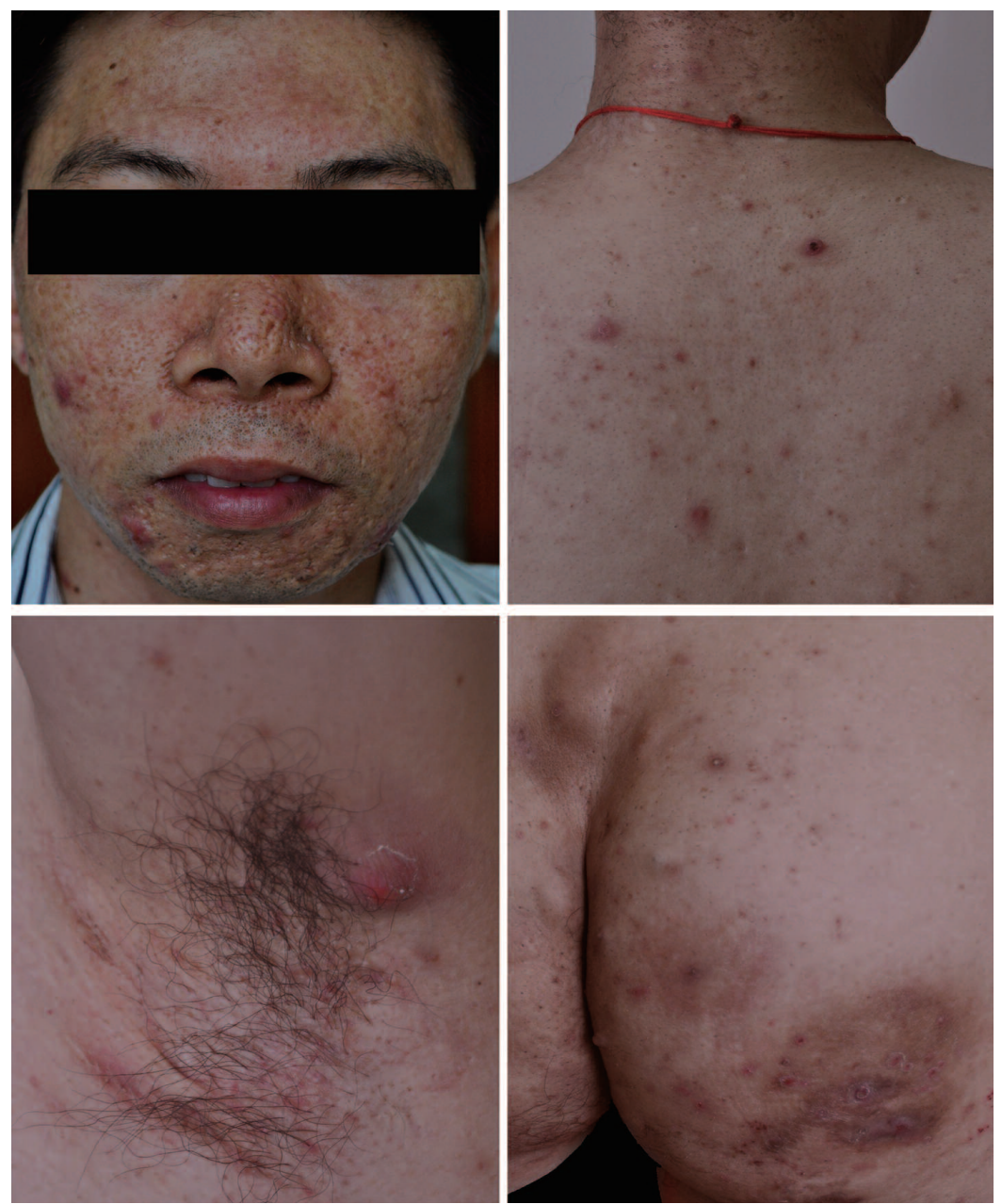

Figure 1. Clinical features of the patient with synovitis, acne, pustulosis, hyperostosis, and osteitis (SAPHO) syndrome. Inflamed papules, nodules, cyst, and scars on the face, nape, back, armpit, and buttocks.

Personal non-commercial use only. The Journal of Rheumatology Copyright $\subset$ 2018. All rights reserved. 


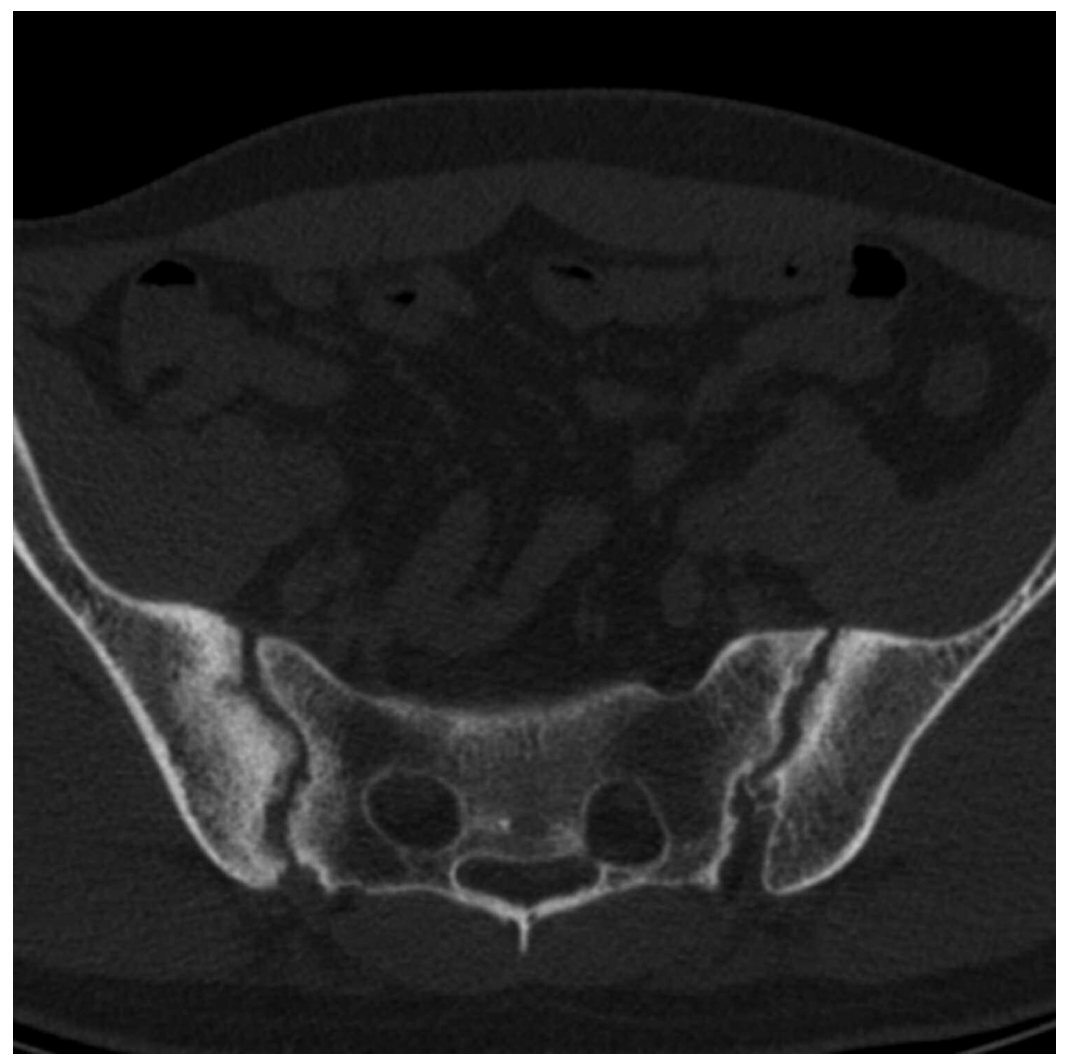

Figure 2. Computed tomography scan shows erosion-like changes in the sacroiliac joint.
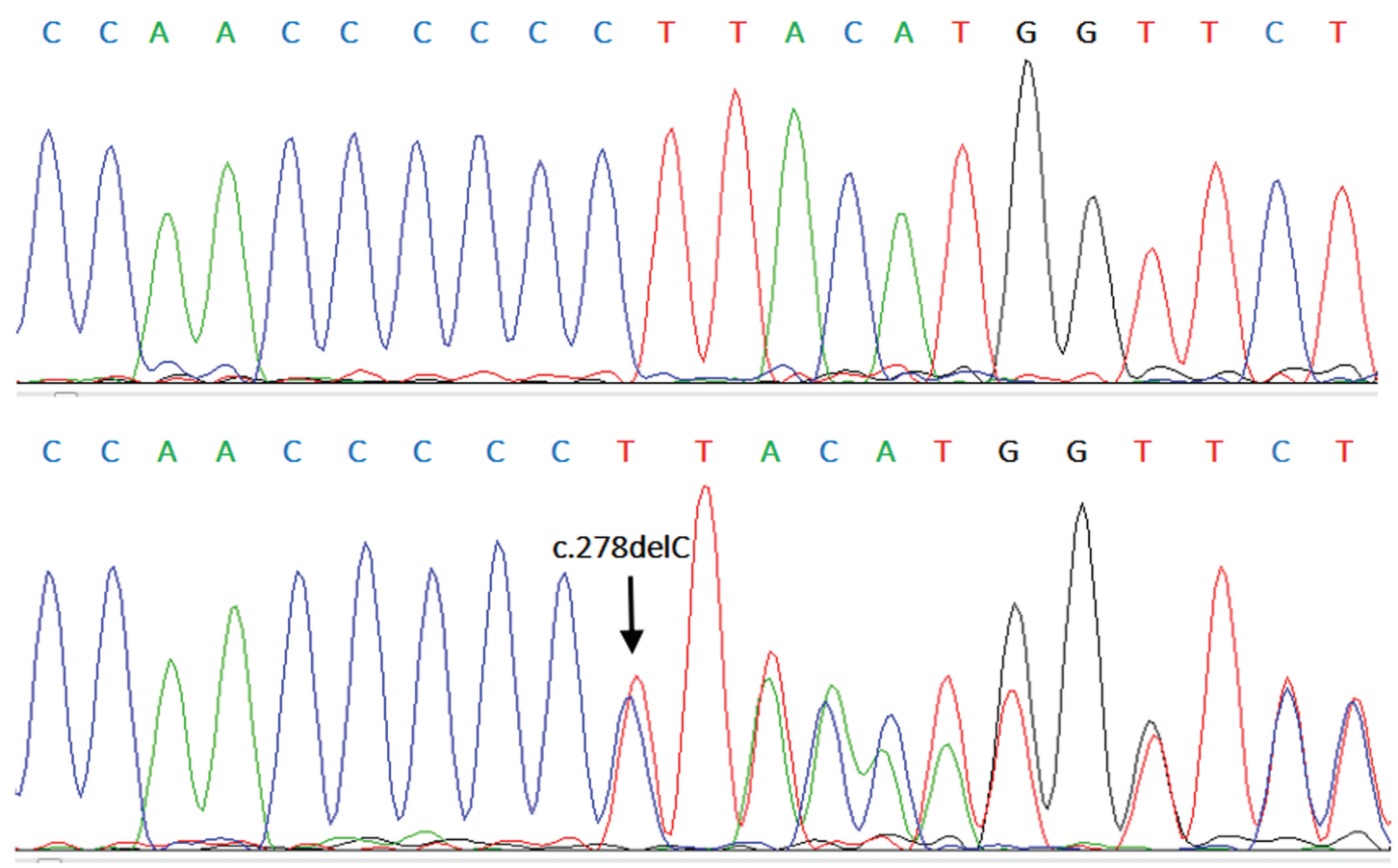

Figure 3. Schematic of the wild-type genotype in unaffected individuals (top panel), and a single-nucleotide deletion mutation of a cytosine at position 278 (c.278delC) of the nicastrin gene (bottom panel), by Sanger sequencing.

Personal non-commercial use only. The Journal of Rheumatology Copyright @ 2018 . All rights reserved. 
mutations, including NCSTN, were found in different patients with PASH syndrome ${ }^{2,7,8}$. We reported that an NCSTN mutation was detected in a patient with SAPHO syndrome, which added a new phenotype to the spectrum of clinical phenotypes resulting from NCSTN mutations, but the relationship between these 3 disorders and NCSTN remains unknown.

SAPHO syndrome and PASH syndrome are both related to HS regarding clinical manifestation, and HS is a clinical manifestation of both. Regarding pathogenesis, the 3 disorders categorized as autoinflammatory diseases are related to large amounts of neutrophil activation ${ }^{7,9}$. With regard to gene mutations, HS, PASH syndrome, and SAPHO syndrome are all linked to the NCSTN gene. As early as 2014, researchers have shown that HS is not solely a skin disease but also a systemic disorder that can be accompanied by other disorders or damage ${ }^{10}$. HS may have genetic backgrounds, environmental factors, or inflammatory pathways in common with these diseases 9 ; the results of our study provide direct evidence to confirm this speculation. Because the clinical presentation of different patients with SAPHO syndrome has obviously different manifestations (i.e., phenotypic heterogeneity), we propose that this syndrome has many pathogenic genes in addition to NCSTN (i.e., gene heterogeneity). In addition, SAPHO syndrome manifesting with HS may be closely related to mutations in gamma-secretase subunits, especially the NCSTN gene. The limitation of our study is that because of the rarity of the SAPHO syndrome, there was only 1 patient; our findings need to be confirmed with additional studies of similar patients. Therefore, we recommend that patients with SAPHO syndrome presenting with HS undergo screening for gamma-secretase mutations. This would reveal whether HS, SAPHO syndrome, and other disorders that manifest with HS (e.g., PASH syndrome) in a group of autoinflammatory diseases have a common genetic mutation background, or HS with systemic involvement could be a subtype of SAPHO syndrome.

\section{ACKNOWLEDGMENT}

We are indebted to Dr. Qu Tao and Dr. Zeng Rong from the Chinese Academy of Medical Sciences-Peking Union Medical College for providing data on patients. We also thank the patients and their family members for participation in our study.

CHENGRANG LI, MD, PhD, Institute of Dermatology, Chinese Academy of Medical Sciences and Peking Union Medical College, and Jiangsu Key Laboratory of Molecular Biology for Skin Diseases and STDs; HAOXIANG XU, MD, PhD, Institute of Dermatology, Chinese Academy of Medical Sciences and Peking Union Medical College, and Jiangsu Key Laboratory of Molecular Biology for Skin Diseases and STDs, Nanjing; BAOXI WANG, MD, Institute of Dermatology, Chinese Academy of Medical Sciences and Peking Union Medical College, Jiangsu Key Laboratory of Molecular Biology for Skin Diseases and STDs, Nanjing, and Plastic Surgery Hospital, Chinese Academy of Medical Sciences and Peking Union Medical College, Beijing, China. C. Li and H. Xu contributed equally to this article. This work was supported by the National Nature Science Foundation of China (grant number 81673087, 81502739, 81472872), CAMS Innovation Fund for Medical Sciences (grant number 2016-I2M-1-003), and Innovation Research on Critical Diseases (grant number 2016ZX320014). Address correspondence to Dr. W. Baoxi, Plastic Surgery Hospital, Chinese Academy of Medical Sciences, Peking Union Medical College, Road Badachu 33, Shijingshan District, Beijing 100144, China. E-mail: wangbx@vip.126.com

\section{REFERENCES}

1. Ratnamala U, Jhala D, Jain NK, Saiyed NM, Raveendrababu M, Rao MV, et al. Expanding the spectrum of gamma-secretase gene mutation-associated phenotypes: two novel mutations segregating with familial hidradenitis suppurativa (acne inversa) and acne conglobata. Exp Dermatol 2016;25:314-6.

2. Duchatelet S, Miskinyte S, Join-Lambert O, Ungeheuer MN, Frances C, Nassif A, et al. First nicastrin mutation in PASH (pyoderma gangrenosum, acne and suppurative hidradenitis) syndrome. Br J Dermatol 2015;173:610-2.

3. Chamot AM, Benhamou CL, Kahn MF, Beraneck L, Kaplan G, Prost A. [Acne-pustulosis-hyperostosis-osteitis syndrome. Results of a national survey. 85 cases]. [Article in French] Rev Rhum Mal Osteoartic 1987;54:187-96.

4. Marzano AV, Borghi A, Meroni PL, Cugno M. Pyoderma gangrenosum and its syndromic forms: evidence for a link with autoinflammation. Br J Dermatol 2016;175:882-91.

5. Hurtado-Nedelec M, Chollet-Martin S, Chapeton D, Hugot JP, Hayem G, Gerard B. Genetic susceptibility factors in a cohort of 38 patients with SAPHO syndrome: a study of PSTPIP2, NOD2, and LPIN2 genes. J Rheumatol 2010;37:401-9.

6. Assmann G, Wagner AD, Monika M, Pfoehler C, Pfreundschuh M, Tilgen W, et al. Single-nucleotide polymorphisms p53 G72C and Mdm2 T309G in patients with psoriasis, psoriatic arthritis, and SAPHO syndrome. Rheumatol Int 2010;30:1273-6.

7. Marzano AV, Ceccherini I, Gattorno M, Fanoni D, Caroli F, Rusmini $\mathrm{M}$, et al. Association of pyoderma gangrenosum, acne, and suppurative hidradenitis (PASH) shares genetic and cytokine profiles with other autoinflammatory diseases. Medicine 2014;93:e187.

8. Marzano AV, Damiani G, Ceccherini I, Berti E, Gattorno M, Cugno M. Autoinflammation in pyoderma gangrenosum and its syndromic form (pyoderma gangrenosum, acne and suppurative hidradenitis). Br J Dermatol 2017;176:1588-98.

9. Vinkel C, Thomsen SF. Autoinflammatory syndromes associated with hidradenitis suppurativa and/or acne. Int J Dermatol 2017;56:811-8.

10. Dessinioti C, Katsambas A, Antoniou C. Hidradenitis suppurrativa (acne inversa) as a systemic disease. Clin Dermatol 2014; $32: 397-408$

J Rheumatol First Release August 1 2018; doi:10.3899/jrheum.171007 